\title{
Missing the (Data) Point? Analysis, Advocacy and Accountability in the Monitoring of Attacks on Healthcare in Syria
}

\author{
Sophie Roborgh \\ Humanitarian and Conflict Response Institute, University of Manchester; sophie.roborgh@manchester.ac.uk
}

\begin{abstract}
Monitoring of attacks on healthcare has made great strides in the past decade, even if improvement in information has not necessarily resulted in changes on the ground. However, important questions on the knowledge production process continue to be under-explored, including those pertaining to the objectives of monitoring efforts. What does our data actually tell us? Are we missing the (data) point? This paper explores several monitoring mechanisms, and analyses the limitations of the data-gathering exercise, affecting the ability of healthcare workers to share their experiences. By drawing on the experiences of those involved in the medicalhumanitarian response in non-government controlled areas in Syria, these dynamics are further brought to the fore, advocating for a more discerning approach in the use of data for such disparate goals as analysis on patterns of attacks (and their implications), advocacy, and accountability.
\end{abstract}

Keywords: humanitarianism, localisation, attacks on healthcare, medical anthropology, witnessing, Syria

\section{Background: Studying Attacks on Healthcare in Syria}

The past years have seen a flurry of efforts to comprehensively understand attacks and their impact by a wide range of actors. The unprecedented attacks in the Syrian conflict in particular constitute a watershed, with Syria arguably forming the best researched example to date. In Syria, attacks on healthcare have gained a systematic character since the start of the conflict (Fouad et al., 2017), even showing an increase after the adoption of UN Security Council resolution 2286 condemning those attacks (UN, 2016).

The violence that Syria's health sector has experienced, especially in non-government-held areas, has had profound consequences. Hundreds of healthcare workers and patients have lost their lives, while the indirect effects in terms of trauma and loss of services continue to affect the population long after the attack has occurred (Fouad et al., 2017). Important insights have been gained in the prevalence of attacks on healthcare (PHR, 2019a; 2019b), the measuring of such attacks (Elamein et al., 2017;
Briody et al., 2018; Haar et al., 2018), healthcare workers' experiences of them (Funk et al., 2018; Center for Public Health and Human Rights et al., 2019) and their views on how it affects healthcare provision (Footer et al., 2018).

Recently, a number of reviews have evaluated monitoring mechanisms of attacks on healthcare. Debuf analyses 'international non-judicial fact-finding and investigative mechanisms' for attacks on healthcare (2017: 6), while Mülhausen et al. (2017) and (the same authors in) Zimmerman et al. (2019) assess the evidence base of our current thinking on attacks on healthcare. I am much indebted to their important insights.

Efforts to study attacks on healthcare cannot be seen in isolation from efforts in monitoring attacks on aid workers in general, for example the Aid in Danger project (2019) and the Aid Worker Security Database (2019). In addition, monitoring efforts in adjacent fields, such as education, are informative for comparative purposes, with Bennouna et al.'s recent study (2018) especially helpful in this regard.

This paper critically reflects on key monitoring mechanisms' set-up and objectives. By drawing on

\section{MANCHESTER} 1824

Manchester University Press 
inclusion and verification criteria, it argues that the analysis of patterns of attacks and implications of violence against healthcare are not necessarily served by the same dataset. It then looks into the manner in which advocacy brings some voices more to the fore than others, raising questions on representation and empowerment. It finally moves on to analyse how judicialisation of monitoring of attacks, the increasing need for the legal 'usefulness' of data to facilitate legal accountability, has affected our data-gathering and analytical purposes. By seeking 'evidence' instead of experiences, we risk overlooking the intrinsic value that providing témoignage ['witnessing'] on attacks has as an act in itself, and how it can silence victims and witnesses in its own (well-intended) manner. Finally, it questions whether these three key goals analysis, advocacy and accountability - are all equally well served in our current monitoring approach, or whether a more discerning one is warranted.

\section{Methodology}

This paper draws on an analysis of scholarly literature, policy documents, media and social media. The analysis is also informed by interviews with 32 individuals, who were predominantly healthcare workers and representatives of organisations active in the medical-humanitarian response in northern Syria. Interviews were conducted in the period January to June 2017, mostly in the Turkish city of Gaziantep, which served as a hub for the humanitarian response in the non-government-controlled areas. Participation was based on a snowballing approach through multiple access points, and respondents took part on the basis of anonymity and confidentiality. Consent was provided, where possible, in writing and otherwise verbally.

\section{Limitations}

The 'Monitoring and Advocacy' section and to a lesser extent the 'Monitoring and Accountability' section draw extensively on a particularly informative interview with a representative from a Syrian organisation. His comments are complemented by insights from other staff.

Furthermore, this study does not claim to provide a comprehensive review of all research efforts that have occurred on attacks on healthcare. There are a number of key organisations engaged in monitoring attacks, starting with the International Committee of the Red Cross (ICRC). Through its Health Care in Danger (HCID) initiative, the ICRC has undoubtedly played an important role in galvanising research, and continues to do so (ICRC, 2019a, 2019b, 2020). However, despite the fact that it continues to monitor attacks internally (ICRC, 2018), it halted the publishing of its tally of monitoring attacks. It also is important to note that within the 201115 monitoring reports, there is limited information provided on verification, data-gathering strategies, etc. (ICRC, 2011, 2013, 2014, 2015).

Many contemporary efforts, meanwhile, have particularly concentrated their efforts on studying the Syrian context, including using it as a testing ground for the development of monitoring mechanisms. Due to its ambition and increased perception as a benchmark in monitoring efforts, I explore the WHO's Surveillance System for Attacks on Health Care (SSA) in particular (WHO, 2018). Additionally, the WHO Turkey Health Cluster ran the Monitoring Violence against Health Care $(\mathrm{MVH})$, 'a standardised electronic data collection instrument and a verification process with anonymous real-time incident reporting' (Elamein et al., 2017: 2279). Elamein et al. (2017) published and analysed its outcomes for the period November 2015 to December 2016, where it was employed in Syria. Examples are also drawn from Physicians for Human Rights' monitoring of Syria (PHR, 2019c), as well as from recent scholarly efforts that developed and/or evaluated different monitoring mechanisms of attacks, most notably Haar et al.'s mobile data-gathering tool that is employed infield (2018). Finally, the paper also looks at the synthesising efforts of the Safeguarding Health in Conflict Coalition (SHCC), to which PHR, WHO's Surveillance System for Attacks on Healthcare, and Haar et al.'s partner organisation, the Syrian American Medical Society, contribute (SHCC, 2019a: 16).

As is the case with ICRC, MSF continues to be an important actor in the agenda-setting on this topic. However, it adopts a specific focus in addressing attacks, focusing on MSF-related facilities, specific attacks and specific locales (MSF, 2019a, 2019b). Due to the absence of a publicly available tally, and in-depth information on data-gathering, MSF has been excluded.

\section{Monitoring and Analysis}

\section{(Supposed) Intentionality: An Attack on Healthcare vs. an Attack Affecting Healthcare}

Monitoring efforts aim to offer reliable data that allows analysis of risk factors, patterns of attacks, and protection strategies as well as attacks' implications (see, for example, WHO, 2018: 4). However, there is little reflection on the question whether analysis of such different features of attacks are served well by the same inclusion criteria for datasets. A key question is when to call an attack an attack.

Monitoring mechanisms take different perspectives on this question. Unsurprisingly, the classification of targeting has been highlighted as particularly difficult to assess in monitoring efforts (see Briody et al., 2018), including within the SSA (Mülhausen et al., 2017). In 
order for an incident to be considered an attack, the WHO looks at two elements: the effects on healthcare delivery (which can be direct or indirect), and the question whether the incident appeared to target the healthcare system or not. An incident is categorised as an attack any time it is considered targeted, or any time it causes direct damage (WHO, 2018: 21). When an attack is considered not to be targeted, and the effects on health service delivery are indirect, the attack is categorised as a 'grey area', requiring 'case-by-case' assessment, to see if an impact on healthcare or element of targeting can be discerned after all (2018: 21).

The SSA offers the following example of an attack within the category not targeted/direct impact: 'a paediatrician is killed while in a market place, unrelated to their role as health personnel' (WHO, 2018: 21). ${ }^{1}$ In this example, the foregrounding of impact over purpose of attack appears based on WHO's definition of an attack: 'Any act of verbal or physical violence, threat of violence or other psychological violence, or obstruction that interferes with the availability, access and delivery of curative and/or preventive health services' (2018: 31, italics added). Essentially, the inclusion of 'interference' suggests that an attack without direct intent to affect healthcare could still be defined as such by virtue of its implications for healthcare. This conflation between attacks affecting healthcare and attacks on healthcare is found consistently throughout SSA's guidelines.

PHR (2019a), the ICRC (2015: 4), and Haar et al. (2018) similarly focus on the healthcare-related nature of the victim/object, instead of intent. Meanwhile, ICRC has taken a more mixed approach, reflecting its evolving thinking on the topic. It distinguished between intentional and unintentional attacks in its initial report (ICRC, 2011), but appeared not to (at least not explicitly) in its interim reports (ICRC, 2013, 2014). In its final report, it refers to intentional and accidental use of physical force' in its definition of violence (2015: 4), ${ }^{2}$ and mentions 'the intent or effect of interrupting the delivery of health-care services' (2015: 5, note 12, italics added). Interestingly, despite the hesitancy of some of its contributing actors on incorporating intentionality, SHCC does make a distinction. Sensitive of the use of contextual data to indirectly suggest intentionality, they distinguish between 'indiscriminate' and 'intentional' attacks (SHCC, 2019a:17, 2019b)

What are the practical repercussions of these positions on intention? Briody et al. argue that since international law obligates the protection of healthcare, intentionality of attacks is of less importance in monitoring attacks; an indiscriminate attack, for example, is just as much an attack as a targeted one (2018: 2). This position makes sense when considering impact of violence on the healthcare system, where it does not matter whether a paediatrician is killed while working in a facility, or when visiting a market - the effect on the health system of the loss of this specific individual being the same.

However, when monitoring is (at least partially) conducted in support of an investigation into the nature and origins of attacks, and the successfulness of mitigation and protection strategies, it becomes vitally important to differentiate between an attack on an (unrecognisable) paediatrician in a marketplace, or at her place of work. Although the former may be an attack affecting healthcare, it is not an attack on healthcare. The perpetrators have engaged in an illegal act nonetheless by killing non-combatants, but they did not necessarily set out to harm the healthcare system, or even failed to protect it by omission. Through the incorporation of attacks affecting healthcare, but not consciously conducted on healthcare, our analysis on patterns may become muddled through false positives. This affects our specific understanding of the pattern of attacks employed during the conflict against the healthcare system, and brings us more into the territory of the general impact of conflict on healthcare.

However, this analytical distinction between attacks on healthcare and those affecting healthcare is further complicated by the fact that targeting and impact are linked in practice. Perpetrators arguably adopt an array of instruments to accomplish an objective, in this case usually the discouraging, diminishing, or even complete cessation of healthcare provision. The impact of their (previous) attacks on the healthcare system (whether intentional or indiscriminate) is likely to inform their policy of continuing and directing attacks.

\section{Non-random Exclusion in Reporting and Verification Strategies}

Protocols of measuring attacks, especially reporting and verification criteria, also have important implications for analysis. The monitoring mechanisms take different positions pertaining to those actors considered capable of reporting and/or verifying incidents. Part of this is the value attributed to secondary versus primary reports, with the SSA, the ICRC, and PHR willing to accept secondary data, while the WHO Turkey Cluster and Haar et al. require primary reports, and even physical presence of the verifying actor at the site of attack.

The WHO's SSA aims to capture 'any and all attacks' (2018: 5), but pairs their relatively inclusive approach with strict verification protocols and a certainty-level differentiation method, without reflecting on the potential consequences of this approach (2018: 19). Verification also leads to less incorporation of attacks among other initiatives, as visible among Elamein et al., who report that of 402 incidents, only 158 were considered verified (2017: 2281). Despite assessing that 
even unverified reports are likely true, Elamein et al. still caution against 'reporting bias resulting from the political agendas of some of the reporting sources (which might result in over-reporting)' (2017: 2284).

However, one of the main issues is actually underreporting. There are societies where victims of incidents are unable to contribute their experiences to the public record, for instance for fear of reprisals. Moreover, even when attacks are reported, exclusion and verification occurs in a non-random manner, with some perspectives more likely to be excluded than others (Elamein et al., 2017: 2278; see also Mülhausen et al., 2017; Zimmerman et al., 2019).

The WHO SSA, WHO Turkey Cluster, Haar et al., ICRC, and PHR all highlight the limitations of their approach. They mention their difficulty in accessing certain areas, such as rural areas, due to security concerns and absence of system contributors (WHO, 2018: 19; Elamein et al., 2017: 2278). Reporting on smaller facilities, and on attacks of a less violent, visible nature similarly suffered (Haar et al., 2018: 13). Elamein et al. describe a connection between the type of facility and its verification rate, with attacks on hospitals with trauma centres more easily verified than, for example, incidents involving mobile teams or hospitals without a trauma centre (2017: 2281). Those using open sources, moreover, are also concerned about reporting and selection bias reflecting a diminished sensitivity to less violent forms of interference, to less critical resources, and to less central locations (SSHC, 2019a: 16-19; ICRC, 2011: 11; see also Zimmerman et al., 2019).

This has important consequences for analysis. Inclusion criteria excluding some contributors of data on attacks or strict verification standards may exclude crucial examples, and result in an even less complete overview into the origin and patterns of attacks, underrepresenting specific voices and categories.

\section{Monitoring and Advocacy}

Data-informed advocacy is a familiar occurrence in humanitarian circles. Powers showed how activism and information provision were conceptualised as two sides of the same coin. Activism was considered a guiding value in their information production function, while information was perceived 'as a key component of successful advocacy' (Powers, 2016: 411). In Redfield's study of MSF, he describes this practice as 'an overtly motivated form of scientific research, finding facts in the name of values, in the pursuit of both technical and ethical ends' (Redfield, 2006: 3). He goes on to say that,

[i]n this way, they combine assertions of universalized human sentiment and opinion with those of specific expertise, suggesting a modified relationship with traditions of objectivity and neutrality whereby truth might be proclaimed in open association with a point of view. I call this positioned assemblage of fact and value 'motivated truth' to highlight the overt combination of reason and sentiment that it represents. (2006: 5)

Accuracy plays an important role in this, as it is directly tied to organisational legitimacy. Powers holds that 'the history of NGOs [non-governmental organisations] suggests an assiduous cultivation of such values [accuracy and pluralism] as a response to skepticism about their capacity to put forward credible claims about human rights violations' (2016: 413).

However, the combination of advocacy with datagathering and dissemination may create difficulties. This is especially the case, when in the face of grotesque violence the urge to speak out is felt urgently. Mülhausen et al. already noted the '[conflation of] analytical objectives with advocacy aims' in the case of monitoring of attacks on healthcare (2017: 37; see also Zimmerman et al., 2019: 27). Criticising claims on changes in global rates, Mülhausen et al. state:

\begin{abstract}
Although the problematic nature of methodologies and data collection is widely acknowledged, many sources, including some INGOs [international NGOs] and academics, continue to use unreliable statistics to make advocacy claims. The continued emphasis on (incomplete) quantitative data with weak analytical potential, as opposed to increasing qualitative data through contextual and comprehensive approaches, illustrates how evidence production is driven by political and funding agendas that require measurable figures. (2017: 37)
\end{abstract}

Zimmerman et al. highlight the original aims of datagathering by key humanitarian actors, describing how they sought to address organisational and operational objectives first and foremost, instead of other (analytical or advocacy) objectives, for which the data is less wellsuited (2019: 21-2). They caution against 'conflat[ing] awareness-raising with advanced understanding'. In addition, they question whether the orientation toward advocacy is one that actually will result in action that helps in addressing attacks (2019: 28).

\section{Advocacy and Accuracy}

The Syrian case study is here informative, too. As mentioned above, the following analysis draws in particular on my interview with one representative from a Syrian organisation (henceforth referred to as 'the representative'), with whom this topic was extensively covered. It is also supported by comments from others.

The advocacy that aimed to call attention to and halt attacks on healthcare created dilemmas within the Syrian organisations themselves. Despite the willingness among 
local health staff to engage in documentation, there were considerable tensions at times between the staff under fire on the ground and the staff elsewhere, including concerning publicising attacks. Local staff were worried about the repercussions of publishing certain information, which was not necessarily shared by the advocacy and media staff located elsewhere. Firstly, some pressed their media and advocacy departments not to publicly mention the destruction of a facility, as they were afraid that it would allow the Syrian and Russian governments to know that they had successfully hit their target. Having the knowledge that they had hit the right location, local staff were afraid that they might attack again. In an example of one eastern Aleppo hospital that was hit multiple times, local health staff requested media officers not to publicise the attack, 'partially due to paranoia, partially due to psychosocial issues, partially due to real concerns', as the representative mentioned in our interview. He went on to say that

at some point $[\ldots]$ it became very stressful, our relationship became strained. Afterwards we buried the hatchet, but they were afraid that if you tweet about [the attack] or if you release our report to Reuters, that the Russians will know they hit their target [and think] 'Ok, let's hit them again.'

A similar dispute occurred about the level of destruction and the functionality of the affected medical facilities. There were several reasons for staff to want to obscure the actual extent of damages and the ability of the facility to reopen. Security concerns often led to what the representative described as 'semantics problems'. There were moments where local staff did not want it publicised that a facility was back in operation, out of fear it might be hit again. Media and advocacy staff therefore consciously employed different descriptions that left room for interpretation. However, this created its own difficulties, for example when news about a facility was published by a different party and it did not match with the wellconsidered formulation they had provided. Such moments could raise difficult questions for organisations, including raising doubts on the veracity of their accounts.

The difficulty of setting standards on communication from the safety of Turkey also came to the fore. The representative remarked: 'Internally, we had to keep grilling, and this is unfortunate, because it might have alienated the relationship between [local staff on the ground and us], the trust that has to exist. You know, we are here in Turkey and very comfortable.' It signifies the experience of moral dilemmas among the advocacy and media staff, who on the one hand wanted to disclose the extent of violence their local staff faced, and on the other hand wanted to respect their wishes. The representative stated: 'For us the main concern is the safety of our staff.
Our goal is to amplify their voices. So it is difficult when we know they are wrong.'

Ensuring accuracy was not just a struggle internally, it was also a topic of dispute with other local health organisations who struck a different balance. As the representative commented about some other organisations:

They don't care for the media narrative. They don't care about their credibility, they only care about their staff on the ground. And the difference between [us] and [them] is that the leadership [here] has an understanding of the need for credibility. [...] The other NGOs, are not as honest, not as adept in this whole operation, and they played a role that was negative, and non-humanitarian. Although I understand it one hundred per cent. And this is where it gets complicated.

\section{The Selection and Policing of Accounts}

Another issue was the highlighting of some accounts, and the policing of others. In addition to statements by NGO advocacy staff, individual healthcare workers in the field regularly featured in advocacy material and media reports to comment on the situation on the ground. Such forms of witnessing were encouraged by some organisations. As the representative explained, they selected those employees 'that speak English, are media-savvy and don't say stupid [expletive]; don't say something about the revolution or talk too much about Iran or Shi'a'. Some health workers who met these requirements became well-known figures, who regularly featured in advocacy efforts. This, of course, also reinforced notions of a 'deserving' victim and a 'good' humanitarian, one that is politically moderate and palatable for Western consumption.

In addition, the potential PR repercussions of staff members' behaviour outside of working hours caused many organisations to set up informal rules. As the representative pointed out:

We fire people, in the middle of the [Aleppo] siege, for stupid Facebook posts that are political, that might be aligned with groups that are not so savoury. [...] You know, you are a humanitarian. And then also for PR, it would [expletive] us completely if this is [happening].

Other organisations similarly confirmed that they were careful, for instance by asking their employees not to mention their employer on the social media accounts they used for private expression of opinion. One INGO mentioned how they were informally contacted by local authorities about some of the social media statements of their volunteers (interview with INGO, 2017). An important factor in the careful expression of medical-humanitarian organisations were the intense smear campaigns by pro-Assad forces conducted against health organisations and first- 
response organisations that tried to detract from their activities and change the narrative of the conflict (see The Syria Campaign, 2017).

Zimmerman et al. (2019) describe how media has affected the manner in which we perceive attacks on healthcare, in a move toward the extraordinary. Again, this limits our perspective, away from the ordinary, the dreary, the continuous to the more extreme examples of interference with healthcare (see Fast, 2014). The amplification of some voices and experiences (and healthcare workers) over others also affected daily research practice. When approaching Syrian organisations for my study, suggestions for participants were sometimes accompanied by a brief summary of their difficult experiences. Some potential participants appeared to be selected predominantly on the basis of their extraordinary experience and ability to cultivate affect. One media officer, who helpfully forwarded me to several participants, suggested one of them to me by mentioning the specific personal losses the interviewee had suffered while working in a field hospital, and well-known cases they had been involved with. ${ }^{3}$ Hierarchies of suffering were hence created in the facilitation of my research project, perhaps unconsciously so.

At times the networks were overwhelmed with information requests. Some media staff tried to moderate the enormous confusion around the Aleppo siege by creating a WhatsApp group directly connecting top journalists to local actors on the ground, many of whom were selected health workers. Numbering about 267 participants, the WhatsApp group became a key platform, which sought to curb the stream of unsubstantiated rumours. Here, medical-humanitarian organisations positioned themselves as brokers.

The relations between local humanitarian organisations' teams and journalists extended further. The representative stated: 'We are all FB [Facebook] friends. It's a community' (see also Zimmerman et al., 2019: 23-4 on the 'symbiotic relationship' between humanitarian organisations and media). However, the difference in objectives between journalists and organisational representatives created tension. When several well-known journalists travelled to government-held territories in Syria, it was not looked upon favourably. 'We made sure that not many [Syrian] people knew about that. The Syrians, they would never trust [them again] ... They would feel very betrayed about that', the representative stated.

The relationship between data collection on attacks and advocacy is hence anything but straightforward, and moderated by a number of concerns. Practically, it limits access to the perspectives of healthcare providers who are angry, political and unwilling to compromise their perspectives to the measured lingo associated with humanitarianism. When some healthcare workers referred to a 'genocide' in their statements on Aleppo, their perspective was muted by their organisations on the basis of the international legally, politically and historically contentious connotations of the term (interview with 'the representative'). In doing so, this expression was assessed on the basis of its degree of accuracy and the potential inconvenience it might cause, where it fell short, instead of being appreciated as an inherently valuable speech act that attempted to connect a besieged, isolated city in 2016 Syria to a broader world history and global community.

\section{Monitoring and Accountability}

Many of the strategic choices in the constitution of the mechanisms mentioned above, are informed by the objectives the monitors have in mind. Several organisations and scholars highlight the multiple objectives this data has, such as Elamein et al., who state:

It has long been recognised that robust data are crucial to verifying attacks on health care, quantifying their effect (in terms of damage to health structure, staff, and lost services), convincing global actors of the urgent need to enforce international protections for health care, and achieving accountability for breaching them.' (2017: 2279)

Analysis (of both pattern and impact), advocacy and accountability all need to be served by the data such mechanisms would generate. However laudable, the question is, are we able to do all these goals equal justice on the basis of our current approaches? Are they even compatible? What does our data actually tell us? Even when individual monitors highlight the restrictions of their data, they are still often uncritically employed by others in analysis and advocacy (Zimmerman et al., 2019).

A similar conflation as seen with advocacy, occurs with the gathering of data to serve as evidence to inform investigations for judicial proceedings. Law has become a key element informing monitoring, with Haar et al.'s monitoring tool, among others, 'using questions centered on international human rights and humanitarian law' (2018: 4). This is also visible in UNSC resolution 2286 and supporting guidance on implementation, which emphasises investigations and fact-finding, and also supports comprehensive data-collection (Debuf, 2017: 5). This focus informs the data-gathering and verification processes as well, with the same humanitarian organisations involved in all three endeavours of analysis, advocacy and accountability. The aforementioned representative explains how incident monitoring processes in his organisation are driven by a quest for justice:

To be able to prove [a violation] at the UN stage, to be able to have reliable information that in the future can be used for prosecution, you know that is very important to us. It is about justice for our staff on the ground and for these innocent civilians who are denied healthcare, whose lives are getting destroyed and so on and so forth. 
This affects the monitoring efforts profoundly. $\mathrm{He}$ mentioned: 'Even if the director of an [organisation's] Aleppo field team saw a barrel bomb fall on a hospital, we don't care about his testimony. We care about [staff], who are trained in methodology, because we have to follow that protocol.' He continued: 'Specifically on issues such as attacks on health care, talking about documenting war crimes, there are of course even more strict protocols to follow.' Hence, quality is emphasised over quantity. Another representative remarked: 'We don't need one [more piece of] evidence that those attacks are happening. We just need to analyse the data that we know are true. [...] Whether it is 100 attacks or 120, it means nothing. At the end, one attack [on a health facility] is a crime' (interview with a Syrian NGO representative, 2017). The phrasing employed here, 'documenting war crimes', 'evidence', clearly highlights how instead of merely looking for data, there was a search for evidence that would meet the standards necessary to stand up in court. Recollections that fell short of this standard, or came from people who have not undergone the right training for formal verification, did not suffice.

Accuracy was key in their monitoring efforts, not only for purposes of securing legal accountability, to which we will return shortly, but also for reputation. As the representative stated: 'Any wrong reporting can damage the credibility of the organisation indefinitely.' The difference in positionality of Syrian organisations (both grass roots and diaspora ones) and international actors is worth pointing out here. WHO has no qualms about the potential (initial) incorporation of false positives in its effort to count all attacks, allocating differing levels of 'certainty' to incident reports (2018: 19). Syrian organisations, however, are active in a highly politicised environment, where the attacks they report and the subsequent relief efforts they are involved in, are regularly dismissed as 'fake news' by political opponents. They cannot afford the publication of information, which might later be disproven, without potentially incurring significant reputation damage, harming not just their organisation, but the medical-humanitarian response at large.

NGO representatives, desperately seeking to protect their staff and the embattled local population they serve, conduct these efforts with the best of intentions. However, these legally oriented approaches, however understandable, come at a cost. Debuf already argued that many attacks on healthcare do not meet the threshold of 'serious violations of international humanitarian or human rights law'. She argues that 'all incidents of violence or threats against, damage to, or undue interference with the provision of healthcare in armed conflict should be investigated regardless of whether they amount to violations of the law' (2017: 9, italics in original). In practice, foregrounding the legal usefulness of reports obscures the true number of attacks and their costs, decreasing their value for analysing the nature and patterns of attack, as elaborated above. The fear of the repercussions for false positives lead organisations to dismiss a significant amount of highly likely, but not stringently verified, positives.

Such approaches contribute to the creation of 'hierarchies of attacks' (Mülhausen et al., 2017: 38) based on the identity of the reporter, whether verification can be accomplished, and whether the event in practice meets a certain threshold of illegality and seriousness. In order to evaluate the evolution of the comprehensive pressure on the system over time in a specific context, a contextualised approach is needed, which looks at how visible and invisible, incidental and continuous, and legal and illegal instruments are employed in unison in diminishing healthcare provision.

The focus on accountability raises ethical questions as well. As Debuf already pointed out in her reviews, with a few exceptions, 'none of the mechanisms examined for this project seem to be exclusively or even primarily geared toward addressing the needs and grievances of victims of violations of international humanitarian or human rights law'. Although accountability, justice and prevention benefit victims, 'some needs of victims go beyond prevention and accountability, and the mechanisms evaluated generally address these needs less, if at all' (2017: 11). It is, for example, telling that the UN Secretary-General Recommendations on resolution 2286 mention '[p]roviding reparations and assistance to victims and restoring essential services' only as their final point (UN Secretary-General, 2016: 10, Recommendation 13).

It also overlooks the fact that incident accounts have value in themselves for those providing them. Currently, contributors' accounts can be excluded for failing to meet the (externally imposed) threshold, even though it is concern for the lives of these same healthcare workers and the broader population that drives this monitoring strategy. However, accounts of incidents are not just a means to an end, they are an end in themselves. By reflecting a victim's lived experience, they become important accounts of speaking out and témoignage, processes that have intrinsic value. The act of witnessing, for example, bears witness to something 'beyond recognition', and constitutes a deeply personal experience (Oliver, 2001). Unequal accessibility to the witnessing process, in this case mediated through monitoring mechanisms or gatekeeping, perpetuates hierarchies of credibility and visibility, and ultimately, humanity (see Fassin, 2007). Victims are informed by external actors, including in some cases by their own colleagues, that their account is not fit for purpose and falls short of a technical threshold attached for a higher goal. An opportunity for reflection, and (re)constitution of subjectivity is foreclosed in the process.

\section{Conclusion}

Attacks on healthcare continue to plague healthcare workers and their patients in numerous settings, and need 
to be better understood. This paper engaged with two crucial, interrelated questions concerning the data gathered for this purpose: what is this data aimed for, and what does it actually reflect? Building on work by Zimmerman et al. (2019), this paper explored three objectives of datagathering on attacks on medical infrastructure: analysis, advocacy and accountability. It showed how data gathering and dissemination on attacks on healthcare can sit uneasily with these different objectives, individually. I argued how analysis on patterns and origin of attacks and on implications of violence against healthcare is not necessarily best serviced by the same data-collection and verification protocols. I then looked into the relationship between data-gathering and advocacy, highlighting the pressures and difficulties affecting communication between field staff and their representatives located in safer locales. I emphasised the challenging political circumstances in which these organisations work, and the difficult balance their staff must strike. I also analysed the consequences of this balancing act, particularly how some voices were amplified over others, reinforcing pre-existing notions of 'deserving' sufferers and 'good' humanitarians. I subsequently explored the increasing incorporation of accountability objectives into data-collection, showing how this has profound implications for the understanding of these attacks, as well as for victims to be able to express themselves, and re-establish their agency.

That leaves us with the final question on whether all these objectives can be collectively facilitated by the same datagathering and dissemination methods, as is now sometimes aspired to. This paper highlighted the unique, individual, challenges that objectives of analysis, advocacy and accountability bring to gathering and engagement with data on attacks. Although often executed to a certain extent by the same teams, these objectives engage very differently with the monitoring of attacks. If we want data suited to these different objectives, including the oft-overlooked objective of witnessing as a valuable activity in itself, a critical perspective on our current monitoring practice is needed as well as practical steps to rectify shortcomings.

For analytical purposes, for example, we could contemplate the creation of dual datasets on incidents. ${ }^{4}$ One could be aimed toward understanding the pattern of attacks. Here a permissive and inclusive approach is taken toward contributors of information, but a discerning look toward inclusion criteria of attacks, excluding attacks that affected healthcare, but were likely not perpetrated on healthcare. The other could concentrate on the implications (outcomes) of attacks on healthcare. Irrespective of whether these attacks targeted healthcare (i.e. with intention), they have affected the sector both directly and indirectly, requiring permissive inclusion criteria on both contributors or data and attacks. Analysing these facets separately at first will allow a more complete picture to emerge.

\section{Acknowledgements}

I would like to thank the research participants for their time and involved participation. Laetitia Atlani-Duault gracefully provided comments on earlier drafts and guidance for further exploration. Gratitude is also owed to the editors of the special issue, and anonymous reviewers. An earlier draft was presented at the 'Violences Extrêmes - Enquêter, secourir, juger - Syrie/ Rwanda/RDC' conference, organised by Fondation Maison des Sciences de l'Homme and CRASH. I thank organisers, other presenters and attendees for their helpful comments there. All remaining errors are my own. The study was funded with grants from the Economic and Social Research Council (No. ES/ N015754/1, for 'Medical neutrality in context') and from the Isaac Newton Trust. Ethical approval was obtained from the Departmental Research Committee of the Department of Politics and International Studies and the Humanities and Social Sciences Research Ethics Committee (HSS REC) at the University of Cambridge.

\section{Notes}

1 Haar et al., for example, provide an example included in their tally of a physician 'killed by a landmine explosion' (2018: 8). It is not clear from their description whether one can speak of specific targeting in this instance, as an indiscriminate device was employed. Its specific location might shed light on this. Meanwhile, in their study of monitoring attacks on education in the contexts of Somalia and DRC, Bennouna et al. similarly had to contend with this issue: 'Incidents were not admitted when a student or educator was attacked outside of the educational context. For instance, if an armed group assaulted a teacher at home, it was not considered to be an attack on education unless the informant had a convincing reason to believe that the motive was to disrupt education, as with al-Shabaab's series of targeted threats and attacks on school personnel in Mogadishu' (2018: 319).

2 It takes a more intentional definition of violence in its 2011 report (ICRC, 2011: 5).

3 Several points of access were hence employed to secure a more representative sample.

4 Or, alternatively, instead of engaging with datasets where decisions on inclusion have already been taken, raw datasets could be used that leave it to the analyst to set out criteria for inclusion or exclusion. This would facilitate the creation of tailor-made datasets that can answer specific research questions.

\section{Bibliography}

Aid in Danger project (2019). http://insecurityinsight.org/projects/aidin-danger (accessed 11 July 2019). 
Aid Worker Security Database (2019). https://aidworkersecurity.org/ about (accessed 11 July 2019).

Bennouna, C., van Boetzelaer, E., Rojas, L., Richard, K., Karume, G., Nshombo, M., Roberts, L. and Boothby, N. (2018), 'Monitoring and Reporting Attacks on Education in the Democratic Republic of the Congo and Somalia', Disasters, 42:2, 314-35, doi: 10.1111/disa.12245.

Briody, C., Rubinstein, L., Roberts, L., Penney, E., Keenan, W. and Horbar, J. (2018), 'Review of Attacks on Health Care Facilities in Six Conflicts of the Past Three Decades', Conflict and Health, 12:1, doi: 10.1186/s13031-018-0152-2.

Center for Public Health and Human Rights et al. (2019), 'Reality Makes Our Decisions': Ethical Challenges in Humanitarian Health in Situations of Extreme Violence: Report and Recommendation. www.jhsph.edu/research/ centers-and-institutes/center-for-public-health-and-human-rights/ _pdf/Len\%20Violence\%20Report.pdf (accessed 11 July 2019).

Debuf, E. (2017), Evaluating Mechanisms for Investigating Attacks on Healthcare, International Peace Institute (New York), December 2017. www.ipinst.org/wp-content/uploads/2017/12/1712_Attackson-Healthcare-final.pdf (accessed 11 July 2019).

Elamein, M. et al. (2017), 'Attacks against Health Care in Syria, 2015-16: Results from a Real-Time Reporting Tool', The Lancet, 390:10109, 2278-86, doi: 10.1016/S0140-6736(17)31328-4.

Fassin, D. (2007), 'Humanitarianism as a Politics of Life', Public Culture 19:3, 499-520, doi: 10.1215/08992363-2007-007.

Fast, L. (2014), Aid in Danger: The Perils and Promise of Humanitarianism (Philadelphia: University of Pennsylvania Press).

Footer, K. H. A., Clouse, E., Rayes, D., Sahloul, Z. and Rubinstein, L. S. (2018), 'Qualitative Accounts from Syrian Health Professionals Regarding Violations of the Right to Health, including the Use of Chemical Weapons, in Opposition-Held Syria', BMJ Open, 8: e021096, doi: 10.1136/ bmjopen-2017-021096.

Fouad, F. M. et al. (2017), 'Health Workers and the Weaponisation of Health Care in Syria: A Preliminary Inquiry for The Lancet-American University of Beirut Commission on Syria', The Lancet, 390:10111, 2516-26, doi: 10.1016/S0140-6736(17)30741-9.

Funk, K. L., Rayes, D., Rubinstein, L. S., Diab, N. R., Singh, N. S., DeCamp, M., Maziak, W., Ho, L. S. and Robinson, W. C. (2018), 'Ethical Challenges among Humanitarian Organisations: Insights from the Response to the Syrian Conflict', in Ahmad, A. and Smith, J. (eds), Humanitarian Action and Ethics (London: Zed Books), pp. 133-45.

Haar, R. J., Risko, C. B., Singh, S., Rayes, D., Albaik, A., Alnajar, M., Kewara, M., Clouse, E., Baker, E. and Rubenstein, L. S. (2018), 'Determining the Scope of Attacks on Health in Four Governorates of Syria in 2016: Results of a Field Surveillance Program', PLoS Med, 15:4, e1002559, doi: 10.1371/journal.pmed.1002559.

ICRC (2011), Health Care in Danger - A Sixteen-Country Study, July 2011. www.icrc.org/eng/resources/documents/report/hcid-report2011-08-10.htm (accessed 21 January 2020).

ICRC (2013), Health Care in Danger - Violent Incidents Affecting Health Care, January to December 2012, May 2013. https://reliefweb. int/report/world/health-care-danger-violent-incidents-affecting-healthcare-january-december-2012 (accessed 21 January 2020).

ICRC (2014), Health Care in Danger - Violent Incidents Affecting the Delivery of Health Care - January 2012 to December 2013, April 2014. https://shop.icrc.org/health-care-in-danger/health-care-in-danger-violent-incidents-affecting-the-delivery-of-health-care-january-2012-todecember-2013.html (accessed 11 July 2019).

ICRC (2015), Health Care in Danger - Violent Incidents Affecting the Delivery of Health Care, January 2012 to December 2014, April 2015. https://shop.icrc.org/health-care-in-danger/health-care-in-dangerviolent-incidents-affecting-the-delivery-of-health-care-january-2012to-december-2014.html (accessed 15 July 2019).

ICRC (2018), Changing Behaviour: Tackling Violence against Health Care in Niger, the Central African Republic and Nigeria - Selected
Experiences, October 2018. https://healthcareindanger.org/wp-content/uploads/2018/11/4369_002_HCID_selected_experiences_web.pdf (accessed 20 January 2020).

ICRC (2019a), Health Care in Danger project website. http://healthcareindanger.org/ (accessed 10 July 2019).

ICRC (2019b), Health Care in Danger Resource Center. http://healthcareindanger.org/resource-centre/ (accessed 10 July 2019).

ICRC (2020), HCID Initiative. https://healthcareindanger.org/hcidproject/ (accessed 20 January 2020).

MSF (2019a), Attacks on Medical Care website. www.msf.org/attacksmedical-care (accessed 10 July 2019).

MSF (2019b), \#NotATarget website. http://notatarget.msf.org/ (accessed 10 July 2019).

Mülhausen, M., Tuck, E. and Zimmerman, H. (2017), Health Care under Fire: The New Normal?, London School of Economics and Political Science and Chatham House.

Oliver, K. (2001), Witnessing: Beyond Recognition (Minneapolis: University of Minnesota Press).

PHR (2019a), 'Illegal Attacks on Health Care in Syria' (map). https:// syriamap.phr.org/\#/en (accessed 11 July 2019).

PHR (2019b), 'Physicians for Human Rights' Findings of Attacks on Health Care in Syria' (findings as of June 2019). syriamap.phr.org/ \#/en/findings (accessed 11 July 2019).

PHR (2019c), 'Methodology'. syriamap.phr.org/\#/en/methodology (accessed 11 July 2019).

Powers, M. (2016), 'The New Boots on the Ground: NGOs in the Changing Landscape of International News', Journalism, 17:4, 40116, doi: $10.1177 / 1464884914568077$.

Redfield, P. (2006), 'A Less Modest Witness: Collective Advocacy and Motivated Truth in a Medical Humanitarian Movement', American Ethnologist, 33:1, 3-26. http://redfield.web.unc.edu/files/2015/08/ Redfield-Witness-AE-min.pdf (accessed 15 July 2019).

SHCC (2019a), 2018: Impunity Remains: Attacks on Health Care in 23 Countries in Conflict, May 2019. https://reliefweb.int/sites/reliefweb.int/ files/resources/SHCC2019final.pdf (accessed 10 July 2019).

SHCC (2019b), Intentional and Indiscriminate Codebook (version May 2019). http://insecurityinsight.org/wp-content/uploads/2019/05/ 2018-SHCC-Intentional-and-Indiscriminate-Codebook-v-May2019.pdf (accessed 15 July 2019).

The Syria Campaign (2017), Killing the Truth: How Russia is Fuelling a Disinformation Campaign to Cover Up War Crimes in Syria, 20 December. https://thesyriacampaign.org/press/ (accessed 12 February 2020).

UN (2016). Security Council Adopts Resolution 2286 (2016), Strongly Condemning Attacks against Medical Facilities, Personnel in Conflict Situations. Meetings Coverage. SC/12347, 3 May. www.un.org/ press/en/2016/sc12347.doc.htm (accessed 20 January 2020).

UN Secretary-General (2016). Recommendations of the SecretaryGeneral, submitted pursuant to paragraph 13 of Security Council resolution 2286 (2016), on measures to prevent acts of violence, attacks and threats against the wounded and sick, medical personnel and humanitarian personnel exclusively engaged in medical duties, their means of transport and equipment, as well as hospitals and other medical facilities, and to better ensure accountability and enhance their protection. S/2016/722,18. https://reliefweb.int/sites/ reliefweb.int/files/resources/N1626255.pdf (accessed 11 July 2019).

WHO (2018), Attacks on Health Care, Surveillance System for Attacks on Health Care' (SSA), Methodology Version 1.0, WHO, December 2018. Geneva: World Health Organization; 2018. Licence: CC BY-NC-SA 3.0 IGO. https://www.who.int/emergencies/attacks-on-health-care/SSAmethodology-6February2018.pdf?ua=1 (accessed 11 July 2019).

Zimmerman, H. L., Mülhausen, M. and Tuck, E. (2019), 'Attacks on Healthcare in Conflict: Generating Attention in the Modern Information Landscape', Medicine, Conflict and Survival, 35:1, 1242, doi: 10.1080/13623699.2019.1573801. 